\title{
CONSIDERACIONES SOBRE LA ADQUISICIÓN DE L2 A TRAVÉS DE LA PERSPECTIVA SOCIOLÓGICA DE EMILE DURKHEIM Y SU OBRA: LA DIVISIÓN DEL TRABAJO SOCIAL
}

\author{
DIEGO FERNANDO PIÑA PIÑA ${ }^{1}$
}

\section{RESUMEN}

La adquisición de una segunda lengua es un fenómeno que se presenta en un contexto social determinado, en el que las relaciones sociales entre sus miembros desempeñan una labor importante. Emile Durkheim, en su texto, La división del trabajo social (1893) pone de manifiesto dos términos: Solidaridad Mecánica y Solidaridad Orgánica, los cuales serán un eje fundamental en el desarrollo del presente artículo. Este trabajo, también se apoya en las perspectivas de Bourdieu y Vigotsky. Los conceptos de Transdisciplinariedad y Capital cultural, junto a experiencias en el aula, serán elementos que permitirán establecer reflexiones sobre la relación entre la adquisición de una segunda lengua $y$ las nociones sociológicas de Emile Durkheim mencionadas anteriormente, para lo cual se recurrió a una exhaustiva revisión teórica y se contrastaron diferentes posiciones, de tal manera que se le otorgue a los lectores elementos que les permitan adoptar una posición propia.

Palabras Clave: Relaciones sociales, Solidaridad Mecánica, Solidaridad Orgánica, Adquisición de una segunda lengua (L2), Transdisciplinariedad, Capital Cultural

\footnotetext{
${ }_{1}^{1}$ Afiliado a la Universidad Distrital Francisco José de Caldas (Colombia). Cra. 8 \#40-62, Chapinero Bogotá, Colombia. Correo electrónico: blackidion.92@hotmail.com.
} 


\section{CONSIDERATIONS ABOUT SECOND LANGUAGE ACQUISITION THROUGH THE EMILE DURKHEIM'S SOCIOLOGICAL PERSPECTIVE AND THEIR WORK: THE DIVISION OF LABOUR IN SOCIETY}

\section{ABSTRACT}

Second Language Acquisition is a phenomenon that is present in a determinated social context, where social relationships between their members develop an important work. Emile Durkheim in their text The Division of Labour in Society (1893) establishes two terms: Mechanical solidarity and Organic solidarity, which will be fundamental axis in the development of the present article, where perspectives as handled by Bourdieu and Vigotsky, linked to the concepts Transdisciplinarity and Cultural Capital, by the side of experiences in the classroom will be elements used to establish thoughts about the relationship between second language acquisition and Emile Durkheim's sociological notions previously noticed, for that reason an exhaustive theoric inquiry was done and different positions were contrasted, giving to the readers elements that will allow them to adopt an own position.

Key Words: Social relationships, Mechanical solidarity, Organic solidarity, Second language acquisition, Transdisciplinarity, Cultural capital.

\section{Introducción}

La adquisición de una segunda lengua se convirtió en materia de análisis desde mediados del siglo XX. Fenómenos como el creciente intercambio económico a nivel internacional y la globalización, en general, han despertado el interés de los distintos gobiernos por incluir en sus planes de gobierno, políticas de bilingüismo a nivel educativo que permitan un acceso a la población a una segunda lengua desde edades tempranas.

Emile Durkheim (1973), en su obra La División del trabajo social, emplea dos conceptos básicos: Solidaridad mecánica y Solidaridad orgánica, los cuales emplea para dar cuenta de un proceso progresivo que se deriva de la división social del trabajo. En el nivel de integración y especialización de las labores da cuenta del progreso de dicho sistema social. En el aula de clases es común 
observar en los estudiantes una distribución específica, ya sea para establecer algún vínculo social como para realizar alguna actividad académica solicitada por el docente. En este artículo se pretende abordar la adquisición de una segunda lengua desde un ámbito sociológico, atravesado por los conceptos de Solidaridad Orgánica y Solidaridad Mecánica. Para tales fines, se empleará algunos constructos teóricos que permitan dar cuenta de la relación entre lo social y lo académico así como de visiones opuestas a este enfoque. Además, se invita al lector a la reflexión y a la toma de una posición crítica respecto a la temática tratada.

\section{Emile Durkheim y su obra}

Durkheim nació en 1858. Fue un sociólogo y antropólogo francés. Su tesis doctoral y eje central de este artículo "La División del Trabajo Social" es el producto y reflejo de una constante preocupación por la manera en que las sociedades modernas se encontraban cohesionadas (Cohesión Social) (1983). Estableció en su estudio dos categorías fundamentales: Solidaridad Mecánica y Solidaridad Orgánica, las cuales serán abordadas con detenimiento más adelante.

Durkheim también presentó un marcado interés por la educación, la cual define como un fenómeno social. En su obra homónima "La educación como fenómeno social" la define como:

la que nos enseña a dominarnos, a constreñirnos; es también, siguiendo sus necesidades, la que decide la cantidad y naturaleza dc los conocimientos que debe recibir el niño, y lo mismo que es a través de ella que se conserva la ciencia adquirida por las generaciones anteriores, es también la que la transmite a las nuevas generaciones. Es, pues, la que forma en nosotros todo lo que supera la esfera de las puras sensaciones: nuestra voluntad como nuestro entendimiento son moldeados a su imagen. (Durkheim, 1904, p. 1).

\subsection{Solidaridad Orgánica Y Solidaridad Mecánica}

Estos conceptos desarrollados por Durkheim se han convertido en objeto de estudio por la manera en que ofrecen una explicación plausible a la caracterización de las estructuras sociales que han caracterizado a los pueblos en el transcurrir de los siglos. La solidaridad no solo se relaciona a factores de 
Consideraciones sobre la Adquisición de L2 A tRAVÉS de la PeRspectiva Sociológica...

distribución económica; también implica una caracterización del sujeto perteneciente al sistema social. En dicha estructura se pone de manifiesto cada tipo de solidaridad de la que se desprende un tipo de conciencia colectivo o sentido de pertenencia.

En su obra, La división del trabajo social, Emile Durkheim propone estos conceptos como una manera de explicar el nivel de integración de las sociedades, en donde la solidaridad mecánica se ve vinculada a las formas más primitivas; la conexión entre los individuos se obtiene sobre la base de su escasa diferenciación. Se podría decir que es una solidaridad construida a partir de semejanzas y, por lo tanto, de la existencia de pocas posibilidades de conflicto. Por otro lado, el concepto de solidaridad orgánica puede asociarse a aquellas sociedades en donde la diferenciación entre individuos se hace explícita y, como consecuencia de esto, se presenta una recurrencia de conflictos entre ellos, los cuales sólo pueden ser solucionados si hay alguna autoridad que fije los límites y establezca una serie de normas. Respecto a estos tipos de solidaridad, Durkheim (1973) señala "No sólo, de una manera general, la solidaridad mecánica liga menos fuertemente a los hombres que la solidaridad orgánica, sino también, a medida que se avanza en la evolución social, se va relajando cada vez más" (p.181).Para Durkheim (1858-1917), las "sociedades simples" tienen un bajo nivel de diferenciación social o, en otros términos, una limitada división del trabajo en la que sus miembros dependen de lo que todos hacen. Además, comparten una conciencia colectiva que otorga y garantiza la cohesión social y la supervivencia de dicha sociedad. Por otro lado, en las sociedades modernas, aquellas a las que otorga la caracterización de sociedades avanzadas y gran división del trabajo, los individuos son interdependientes (dependen unos de otros para satisfacer sus necesidades básicas). En ellas también existe una conciencia colectiva que les delega una serie de valores o visiones del mundo comunes y compartidas que sirvan como elemento de cohesión social. Dicha conciencia modera las aspiraciones puramente egoístas de los individuos y los mantiene unidos más allá de sus intereses particulares, lo que no implica su supresión plena. 
Para el sociólogo estadounidense Robert K. Merton (2002):

La fuente de la vida social es, según Durkheim, la similitud de las consciencias y la división del trabajo. La primera es más evidente entre las sociedades primitivas, en las que la "solidaridad mecánica», puesta de manifiesto por la ley represiva, prevalece. La segunda es propia de las sociedades avanzadas, en las que se manifiesta una mayor "densidad dinámica» y en las que las reglas jurídicas definen la naturaleza y las relaciones de las funciones. (p.1)

Esta perspectiva teórica cobra valor para él en cuanto el contexto histórico es observable. En otras palabras, la importancia de la obra de Durkheim radica en la concepción de sociedad y del papel de las normas y leyes sociales de aquella época en la que se otorgaron las condiciones que determinaron una manera distinta de ver y hacer sociología. En este punto es importante resaltar estas palabras de Merton (2002) en referencia a su lectura de la obra de Durkheim:

Por otro lado, la división del trabajo social, que si bien intensifica no impone la individualización, también ocasiona una "solidaridad orgánica», basada en la interdependencia de los individuos y los grupos que actúan cooperativamente. Este tipo de solidaridad es indiciada por reglas jurídicas que definen la naturaleza y las relaciones de las funciones. (p. 202)

\section{Funcionalismo Educativo}

Espinoza (2008) (citando a Durkheim, 1964; Parsons, 1949, 1951; Welch, 1985) sostiene que:

Dar una mirada general a los supuestos que guían el accionar del paradigma funcionalista permite concluir que los científicos sociales que adoptan esta perspectiva enfatizan aspectos tales como la relevancia de la integración funcional, el gradualismo, la armonía y la estabilidad. (p.2)

De esta manera, Espinoza (2008) (citando a Davis, 1949; Davis \& Moore, 1945; Easton, 1956; Parsons, 1940) afirma que los investigadores de tradición funcionalista aceptan la inequidad en la sociedad y, a su vez, la ven como una condición necesaria para mantener un orden normativo existente. "Asume que la desigualdad no sólo es inevitable sino que es necesaria y beneficiosa para todos, dado que la supervivencia vivencia individual está asociada a la supervivencia y al bienestar de la sociedad en su conjunto" (p.2). La teoría funcionalista pone de manifiesto la necesidad de establecer una serie de mecanismos que permitan la 
resolución de problemas en un sistema social, denominados como instituciones en las que, por ejemplo, la familia ayuda a resolver el problema del suministro (vivienda, educación) de nuevos miembros. Igualmente, en las que las instituciones económicas se muestran como aquellas cuya función es la de alimentar y vestir a la población (Espinoza, 2008).

Espinoza (2008), en relación a Durkheim $(1956,1964)$, plantea la presencia de tres aspectos que fueron decisivos para el desarrollo de la sociología funcionalista de la educación. En primer término, Durkheim desarrolló la tesis histórica de que las transformaciones en los sistemas educativos eran la consecuencia de cambios económicos y sociales externos en la sociedad considerada globalmente. En segundo lugar, Durkheim también afirmó que las características específicas de las estructuras educativas y sus contenidos culturales guardaban una fuerte relación con las necesidades de la sociedad. Finalmente, como resultado de la transición de una sociedad mecánica a otra orgánica, es necesaria una mayor individualización y esto se refleja en los cambios en la pedagogía y en la organización escolar (Espinoza, 2008).

Las relaciones entre educación y Estado, en el trascurrir del tiempo se han visto sometidas a un mayor interés del Estado colombiano en cuanto a su eficacia y eficiencia. Uno de sus elemento de referencia es su capacidad de producción, lo que se evidencia a través de preceptos como lo son la primacía de los medios ante los fines; la medición, matemática y cálculo sobre la intuición, la lógica y el intelecto por encima de lo emocional, lo teórico y técnico sobre lo reflexivo y lo instrumental y lógico sobre lo intrínseco, por citar algunos ejemplos.

Al respecto, Gibson (Como se cita en Espinoza, 2008) afirma:

En lo que respecta a esta nueva lógica imperante en las relaciones entre Estado y educación, existe cada vez mayor evidencia respecto de la preferencia del Estado por incrementar los niveles de control y poder más que promover la participación y el acceso igualitario a diferentes tipos de recursos. (p.3)

El enfoque funcionalista de la educación se ve reflejado en las concepciones que algunos autores han puesto de manifiesto en sus obras. Como 
lo manifiesta Rafael Feito, docente de la Universidad Complutense Madrid, en su ensayo titulado "Teorías Sociológicas de la educación", al igual que ya hiciera Durkheim, algunos autores con enfoques funcionalistas establecen una analogía del tipo educación-cuerpo humano. En un sistema social, cada parte o cada institución tiene una función (o funciones) determinada, siendo estas partes interdependientes. Por ejemplo, la educación está conectada en diversos modos con instituciones económicas, familiares, políticas y religiosas; Sin embargo, los enfoques funcionalistas reconocen que aquella analogía orgánica no puede ser llevada demasiado lejos. Lo anterior, porque a diferencia del organismo vivo, en el que las células están programadas biológicamente para desempeñar sus funciones determinadas, en la sociedad estas funciones son realizadas por personas que no están "biológicamente programadas" (Feito, s.f.).

Uno de los representantes más importantes del funcionalismo educativo es el sociólogo americano Talcott Parsons (1902-1979), quien propone la revolución educativa como uno de los hechos que permitieron la inmensa extensión de la igualdad de oportunidades. A su vez trae consigo diferencias de habilidad, provenientes del hecho que los individuos son distintos en lo que se refiere a lo que puede hacer. Sus orientaciones familiares y sus motivaciones individuales, ponen de manifiesto variaciones en el nivel de interés y motivación que presentan los alumnos en su deseo de ser aplicados y trabajar duro. Son estas diferencias en el logro educativo las que traen consigo nuevas formas de desigualdad, al ser la certificación educativa la que determina el cargo que se termina por ocupar.

En su famoso artículo "El aula como sistema social" Talcott Parsons (19021979) estudia la escuela en su doble faceta de órgano de socialización y de órgano de distribución de los recursos humanos o de mano de obra. El criterio utilizado por la escuela para hacer esa doble operación es distinto al de la familia: es el criterio del rendimiento. Por este motivo, el mundo de la escuela supone para el alumno una ruptura de las reglas de juego que hasta entonces le habían sido aplicadas. La familia está estructurada sobre la base de elementos biológicos tales como sexo, rango de nacimiento, edad y otros elementos que chocan con las 
reglas del funcionamiento escolar. En este escenario se trata incondicionalmente al niño, que es valorado y querido por el mero hecho de pertenecer a la familia; sin embargo, la escuela anticipa al niño cómo va a ser tratado por la sociedad y su cometido consiste en que el niño internalice esas reglas de juego - que se entrene en su ejercicio - y que compruebe los efectos de su aplicación:

Este trato que por primera vez recibe el niño, puede constituir una fuente de tensiones internas, las cuales pueden resultar aliviadas en virtud de una serie de mecanismos, como son el apoyo familiar, la imparcialidad del profesor, el carácter maternal de la profesora (sic) para con los niños más pequeños y por el trato con el grupo de amigos. (Feito, s.f., párrafo, 17)

Por otra parte, el sociólogo y lingüista británico, Basil Bernstein (1924-2000) manifiesta su preocupación por el fracaso de los estudiantes provenientes de las clases trabajadoras. Este autor realiza una serie de investigaciones empíricas y "desarrolla un instrumental heurístico para comprender la relación entre las funciones en el sistema educativo y la estructura de clases sociales" (Morata, 1996 P. 55). Su objeto de estudio es el aula de clases. Parte de los resultados de su observación, para proponer una teoría capaz de comprender la relación de lo micro y lo macro (el aula y la sociedad).

Como toda teoría, el funcionalismo educativo ha recibido fuertes críticas por parte de los seguidores de la teoría de la reproducción, al considerarla como una teoría que sostiene a la educación como un medio mediante el cual son reproducidas o perpetuadas las relaciones sociales desiguales vigentes. A diferencia de las teorías pedagógicas, que proyectan intervenciones sobre la educación, las teorías educativas de la reproducción se limitan a describir y explicar el funcionamiento de la escuela, y destacan, fundamentalmente, su papel reproductor. Es importante mencionar a las escuelas de ideología marxista como principales opositores pero se profundizará sobre ellos más adelante. 


\section{Adquisición de una Segunda Lengua (L2): Concepciones Teóricas}

Uno de los autores que más ha profundizó en la adquisición de una segunda lengua (L2) es, sin duda, el psicólogo ruso Lev Vygotsky (1896-1934). Desde la perspectiva de Álvarez (2004) para Vygotsky:

el pensamiento y el lenguaje son funciones mentales superiores con raíces genéticas diferentes, tanto filogenética como ontogenéticamente, desarrollándose en una continua influencia recíproca. En este sentido se diferenciaba de las posturas que defendían un "continuismo" entre el intelecto general y los procesos psicolingüísticos. (párrafo 4)

Álvarez (2004) presenta como Vygotsky, en su Modelo de aprendizaje sociocultural, plantea una relación existente entre desarrollo y aprendizaje como dos procesos que interactúan entre sí. El aprendizaje es un elemento que desempeña un rol muy importante al considerarse como un factor del desarrollo. La adquisición de un lenguaje, es visto como un proceso ligado a las distintas formas de socialización de individuo y, en el que el hombre es concebido como una construcción más social que biológica. Las funciones superiores son el resultado del desarrollo cultural en el cual se ve implícita la labor de distintos mediadores.

Lucci (citando a Molón, 2007) sostiene que los intereses de Vygotsky por la psicología tienen su origen en la preocupación por la génesis de la cultura. El hombre es entendido como constructor de la cultura, oponiéndose a la psicología clásica que, desde su perspectiva, no daba una respuesta adecuada a los procesos de individualización y a los mecanismos psicológicos que los generan. Lo anterior, le permite plantear su teoría de la génesis y naturaleza social de los procesos psicológicos superiores: 
Vygotsky se empeñó en crear una nueva teoría que abarcara una concepción del desarrollo cultural del ser humano por medio del uso de instrumentos ${ }^{2}$, especialmente el lenguaje, considerado como instrumento del pensamiento. (Bonnin, 1996, citado en Lucci, 2007, p. 4)

De acuerdo con Bruner (1998), para Vygostky "la mente no crece ni naturalmente ni sin ayuda. No está determinada ni por la historia ni por las limitaciones lógicas de sus operaciones presentes" (p. 8). La inteligencia debe ser vista como la "agudeza" para usar los conocimientos y procedimientos que han sido transmitidos culturalmente como "prótesis" de la mente. Dichos elementos son determinantes en la disponibilidad y la distribución de esos mecanismos de prótesis dentro de un sistema cultural.

La teoría de Vygostky se centró en "caracterizar los aspectos típicamente humanos del comportamiento para elaborar hipótesis de como esas características se forman a lo largo de la historia humana y se desarrollan a lo largo de la vida del individuo" (Vygotsky, 1996 citado en Lucci, 2007, p. 5). Entre los constructos teóricos planteados por Vygotsky encontramos al hombre visto como un ser histórico-social o histórico-cultural, que es moldeado por la cultura creada por él mismo; el individuo se ve determinado por las interacciones sociales, siendo estas la relación con el otro. Aquí el lenguaje sirve como modo para determinar al individuo y es determinante de los otros individuos. En este punto, es importante señalar que "la existencia de diferentes lecturas de la obra de Vygotsky y, por lo tanto, son identificados, por lo menos, dos tipos de determinación del sujeto: la interacción con otros sujetos y el lenguaje" ${ }^{3}$ (Molón (1995) como se citó en Lucci, 2007).

2 "Los instrumentos son medios externos utilizados por los individuos para interferir en la naturaleza, cambiándola y, consecuentemente, provocando cambios en los mismos individuos" (Lucci, 2002, p.140).

${ }^{3}$ De esas determinaciones resulta el sujeto interactivo, es decir, aquel que no es ni activo y ni pasivo, y que es constituido en la y por la relación interpersonal, y el sujeto semiótico, "sujeto constituido en la y por el lenguaje, siendo que apareció como sujeto resultante de la relación (...), y como sujeto constituido en la relación constitutiva EU-OTRO, en una relación dialéctica" (Molón, 1995. p.106). 
En este mismo orden de ideas:

Vigostky sostiene también que el desarrollo mental es, esencialmente, un proceso sociogenético, aspecto a relacionar con la forma en que cultura es interiorizada bajo la forma de sistemas neurofísicos que constituyen parte de las actividades fisiológicas del cerebro, las cuales permiten la formación y el desarrollo de los procesos mentales superiores. (Lucci, 2007, p. 5-6)

Es importante señalar la existencia de una relación entre lo cultural y la adquisición de una lengua, por lo que es posible el establecimiento de otra relación importante: Adquisición de una segunda lengua y la cultura en que ésta se ve envuelta, pero, yendo más allá, resulta pertinente considerar al aula de clases como un ambiente en donde la cultura se pone de manifiesto y es allí donde el concepto de transdisciplinariedad aparece, sirviendo como un puente entre estos conceptos.

Para hablar de transdisciplinariedad es necesario tomar las concepciones de Edgar Morín, filósofo y sociólogo francés, nacido en 1921, quién propone el término transdisciplina como una forma de organización de los conocimientos. Morín hace énfasis en lo que está entre las disciplinas, lo que las atraviesa a todas, y en lo que está más allá de ellas.

La transdisciplinariedad "entiende que el fenómeno objeto de estudio puede ser más completamente comprendido que a través de las vías disciplinares" (De la Herrán, 2011, p. 2). Tomando como base estas concepciones, es necesario comprender aquí de lo que realmente habla este concepto. En lo que concierne a la transdisciplinariedad, se trata a menudo de "esquemas cognitivos" que pueden atravesar las disciplinas, a veces con una virulencia tal que las pone en trance (Morín, 2001). Por otro lado, De la Herrán (2011), sostiene que "si las disciplinas están compuestas de temas, y todos los temas son intrínsecamente transdisciplinares, se puede deducir que cualquier disciplina es íntimamente transdisciplinar. En otras palabras: Si se indaga en la propia disciplina de referencia se puede desembocar en lo transdisciplinar" (p.4). 
El enfoque transdisciplinar del conocimiento y de la educación se puede experimentar de un modo más funcional, menos dual o más sintético y abierto que el disciplinar. Potencialmente, admite y se nutre de más complejidad potencial que lo inter o lo disciplinar, porque en niveles avanzados de desarrollo los pueden incluir. (De la Herrán, 2011, p. 6)

La transdisciplinariedad presenta gran relación con lo social, son las ciencias sociales ${ }^{4}$ quienes ponen de manifiesto, en mayor medida, esta clase de vínculo entre sí. Esta clase de relación, no implica que no se presente entre ciencias que pertenecen a otros campos. Un mayor ejemplo lo encontramos en la educación, en la que podemos encontrar cómo diferentes ciencias se relacionan entre sí, tales como la matemática o el lenguaje cuyo fin esencial es el mismo: formar, instruir y constituir al sujeto. Se podría decir hasta este punto que es la transdisciplinariedad el elemento vinculante que permite establecer relaciones entre lo social y lo educativo, entre el aula de clases y la división social del trabajo.

\section{Concepciones educativas: Durkheim y Bourdieu}

Durkheim y Bourdieu como sociólogos han tenido gran reconocimiento por sus concepciones respecto a la educación. Es observable la diferencia en su visión de ésta e incluso hay autores que se encargan de establecer firmes diferencias entre estos autores y sus perspectivas educativas.

De acuerdo con Morales (2003) "la noción conservadora e idealizada de la educación en Durkheim es denunciada por Bourdieu al situarlo en una posición de "idealismo educativo", que concibe la educación como un proceso realizado por mecanismos institucionales y consuetudinarios". Afirma también, que Durkheim se aleja de estas definiciones de la educación ${ }^{5}$ puesto que las considera

\footnotetext{
${ }^{4}$ El surgimiento de las ciencias sociales como áreas autónomas, bien delimitadas del conocimiento científico, se vincula especialmente al auge del capitalismo europeo y a la emergencia de los procesos económicos, políticos y sociales y sus contradicciones intrínsecas, como esferas claramente delimitadas y secularizadas de la vida y con patrones de comportamiento relativamente estables y empíricamente observables, lo que posibilitó la conversión de estas esferas y sus diferentes dimensiones en objetos de estudio de la ciencia.(Carrizo, Prieto y Klein, 2004)

${ }^{5}$ Morales (2003) menciona aquí que Durkheim refuta el ideal kantiano que propone como fin de la educación el desarrollar todas las facultades humanas, ideal que considera no ser integralmente realizable, puesto que es necesaria la diferenciación social de las aptitudes y de la preparación para actividades diversas, no hay facultades humanas universalmente válidas. Por otra parte rechaza también la definición utilitaria de James Mill, que propone como objetivo de la educación
} 
idealizaciones vanas. Además porque elevan la educación a una suerte de creencia en un patrón de vida estandarizado y universalizable, lo cual se sustenta a través de la siguiente cita:

Parte de este postulado que asegura la existencia de una educación ideal, perfecta, válida para todos los hombres indistintamente; y es esa educación universal y única que el teórico se afana en definir. No obstante, si se considera la historia, no se encuentra en ella nada que confirme semejante hipótesis. (Durkheim, 1975, p.45)

Morales (2003) (citando a Bourdieu y Passeron, 1996) menciona que:

La selección de significados que define objetivamente la cultura de un grupo o de una clase como sistema simbólico es arbitraria en tanto que la estructura y las funciones de esta cultura no pueden deducirse de ningún principio universal, físico, biológico o espiritual, puesto que no están unidas por ningún tipo de relación interna a la naturaleza de las cosas o a una naturaleza humana. (p.4)

Según Morales (2003), para Bourdieu "el pensamiento de Durkheim refleja una visión conservadora propia de una filiación política con la III República Francesa, cuya visión la educación no pretende disminuir y despojar al individuo de sus potencialidades creadoras". Es clave puntualizar que para Durkheim, desde la visión de Morales, el fin de la educación es "convertir el individuo en un ser nuevo, de egoísta-asocial a moral-social", por lo que llega a definir la educación en última instancia como:

La acción ejercida por las generaciones adultas sobre aquellas que no han alcanzado todavía el grado de madurez necesario para la vida social. Tiene por objeto el suscitar y desarrollar en el niño un cierto número de estados físicos, intelectuales y morales que exigen de él tanto la sociedad política en su conjunto como el medio ambiente específico al que está especialmente destinado. (Durkheim, 1975, p. 53)

Gangas (2011), sostiene que Scheler se equivoca cuando compara la Sociología y la Epistemología de Durkheim". La creencia de que Durkheim reduce el conocimiento a una práctica educativa ha sido refutada por muchos académicos (Wallwork 1972; Gieryn 1982; Hund 1990). Frente a esto, Prus Robert (2011)

hacer del individuo un objeto de dicha para sí mismo y para sus semejantes, lo cual le resulta también insatisfactorio puesto que la dicha es un estado subjetivo de las conciencias individuales. 
plantea que "es importante reconocer, con Durkheim, que la manera en que los adultos aprenden y actúan, tiene sus raíces en la educación infantil".

La educación en Durkheim en definitiva puede verse asociada con una "des-idealización", pero, a su vez es asociable al concepto de solidaridad, en la que un nivel de integración mayor implicaría un mayor desarrollo social. Si esto se traslada al aula, puede entenderse a través de un concepto al que denomino como Solidaridad en aula, en donde el nivel en que las relaciones sociales presentan un nivel mayor de integración determinarían la eficacia de actividades aplicables por parte del docente en lo que respecta a la adquisición de una segunda lengua (L2), aspecto a profundizar más adelante.

\section{Capital Cultural en el Aula}

Para hablar de capital cultural, es necesario recurrir a autores cuyos estudios les ha permitido establecer concepciones claras y puntuales. Teresa Bracho es una docente mexicana con un Doctorado en Ciencias Sociales con especialidad en Sociología, quien define capital cultural como la herencia social y cultural que desempeña un importante papel en procesos de selección y exclusión sociocultural. "Incluye todos los recursos culturales transmitidos en la familia, los hábitos de comunicación e interacción aprendidos en ella y los signos culturales asociados a status alto, reconocidos y legitimados como tales". (Bracho, 1990, p.3).

Para Bourdieu (1980) el capital cultural puede tomar tres formas: bajo el estado objetivado, el estado institucionalizado y el estado incorporado. El estado objetivado se refiere a objetos con propiedades que ejercen por su sola posesión un efecto educativo en los agentes. El estado incorporado se refiere al trabajo realizado sobre el cuerpo, que se observa en hábitos, esquemas de percepción y gusto. El estado institucionalizado se refiere a los certificados que la escuela (y otras instituciones) otorga como reconocimiento del desarrollo de un conjunto de habilidades escolares. 
La importancia del capital cultural radica en su función como un elemento que nos permite dar cuenta del recorrido académico de los estudiantes en el aula de clases. Esto resulta de gran importancia si nos interesa conocer el nivel de acercamiento que dicho grupo de estudiantes presenta en relación a una segunda lengua, asimismo del acercamiento que presenta respecto a la cultura de la que dicha lengua hace parte. Por otro lado, puede ser tomado como un punto de partida del establecimiento de hipótesis que permita dar cuenta del porqué de situaciones en la que los estudiantes, pese a poseer un bajo capital cultural, presentan un desempeño académico elevado.

En cuanto al acceso a la educación superior, un capital cultural elevado, a simple vista, implicaría un mayor acceso a la participación en estos procesos. Respecto al tema, De Garay (2001) (como se cita en Colorado, 2009) afirma que "los jóvenes que logran ingresar a las instituciones de educación superior no son un grupo homogéneo, provienen de diferentes estratos sociales, ambientes culturales y familiares y tienen diferentes expectativas e imaginarios sobre la educación superior" (p. 1). Por su parte, Colorado (2009) sostiene que "el tránsito por un sistema educativo que tiende a ser cada vez más cerrado los pone en situaciones diferentes a las cuales deben enfrentarse dentro de la universidad y para lo cual deben hacer uso de diferentes recursos" (p. 1). De esto se desprende que un buen desempeño escolar puede verse afectado por factores de diversa índole, tales como el "origen social", el capital cultural, la motivación personal o el apoyo familiar.

\section{Críticas al Funcionalismo Educativo}

El funcionalismo educativo ha recibido fuertes críticas. Una de ellas delimita al ser humano como un mero producto básico del proceso de socialización, cohibiendo de cierta manera su creatividad, al limitarse a favorecer los intereses del sistema social al que pertenece. Feito (s.f.) hace referencia a la denominada como la "nueva" sociología de la educación, planteada como una reacción frente a la omisión del estudio del curriculum por parte de la sociología. De este modo, el 
Consideraciones sobre la Adquisición de L2 A tRAVÉS de la PeRspectiva Sociológica...

curriculum aparecía como algo externo y separado. Durante el periodo de reorganización educativa de los años 50 y 60, se empezó a argumentar que el dilema básico de nuestro tiempo presenta un carácter cultural y afecta la naturaleza de los significados transmitidos en las escuelas. Basil Bernstein es uno de los principales exponentes de esta escuela, quién tiene como principal objeto de su objeto al aula.

Los enfoques marxistas, por otro lado, consideran que "el sistema educativo es una institución que favorece a las clases sociales privilegiadas, de modo que la escuela se convierte en una institución que justifica las desigualdades previamente existentes" (Feito, s.f.). Otra de las grandes críticas que recibe el funcionalismo proviene en gran parte de Bourdieu y Passeron (como se explicó anteriormente). Retomando a Morales (2009), para Bourdieu, "la educación en realidad lo que crea es desigualdad en el acceso al capital cultural, que se reproduce por el origen social de los individuos", por lo que procede a remitirse el texto Los Herederos, en los que se postula:

Así, hay que distinguir entre la facilidad para asimilar la cultura transmitida por la escuela (mucho mayor a medida que sube el origen social) y la propensión a adquirirla que alcanza su máxima intensidad en la clase media. Aunque el deseo de ascenso a través de la educación no sea menos intenso en las clases inferiores que en las medias, sigue siendo irreal o abstracto dado que las posibilidades de satisfacerlo son ínfimas. (Bourdieu, Passeron, 2004, p. 39)

Martín (2003), por su parte, plantea que "los sociólogos marxistas y críticos, consideran la educación como un instrumento de las clases dominantes para perpetuar la dominación capitalista". En todos los casos, la escuela es vista como un instrumento que cumple funciones para el mantenimiento de la totalidad integrada que es el sistema social. En palabras de Martín (2003), para los funcionalistas:

el sistema escolar sería una pieza esencial en el mantenimiento o perfeccionamiento de la dominación de clase cuya función sería el control y la reproducción social y de conciencias para reproducir el orden capitalista, el sistema de clases, la dominación de la clase dominante, etc. (p. 5) 


\section{Percepción de Solidaridad en el Aula de Clase}

Como se ha mencionado previamente, el concepto de solidaridad propuesto por Durkheim permite dar cuenta del nivel de integración social. Esto puede ser aplicado al aula en tanto ésta puede ser concebida como una "pequeña sociedad", en donde el sujeto pasa a ser formado e instruido para que después pueda aplicar lo aprendido a la sociedad en la que se encuentra inmerso.

En nuestra experiencia en el aula, se pudo percibir cómo las distintas maneras de organización que presentaban los estudiantes afectaban su desempeño en las distintas actividades ${ }^{6}$, las cuales involucraban al contexto social en el que se encontraban inmersos. Ello en relación con el contexto en que la segunda lengua (L2) sería aprendida naturalmente. Posterior a esto, se procedió a observar su desempeño en dichas actividades tanto individual como grupalmente, lo que permitió establecer una serie de conclusiones, las cuales consideramos pertinentes consignar en este artículo.

Las actividades fueron realizadas por separados en los grados 801 y 1102 de la jornada tarde en la Institución Educativa Silveria Espinosa de Rendón, ubicada en el sector de Puente Aranda, en la ciudad de Bogotá (Colombia), durante el año 2014. En el grado octavo se pudo observar, respecto a las actividades grupales, que quienes se encontraban en grupos con personas con quienes poseían buenas relaciones sociales (Los estudiantes que conformaron los grupos se seleccionaron por orden de lista) participaban más en actividades que involucraban movilizarse del salón o pasar al tablero. No obstante, su desempeño en las actividades era más bajo en relación con quienes se encontraban en grupos con personas con las que presentaban un bajo índice de integración respecto a los miembros de su grupo. El desempeño (reflejado en notas) de los estudiantes en general, era más alto cuando trabajaban en grupo que a nivel individual, en los

\footnotetext{
${ }^{6}$ Mufioz, Lopez. (2003) señalan que es importante que las actividades que se planteen sean motivadoras y estén conectadas con su mundo, para mantener el interés inicial y poder desarrollar un aprendizaje significativo. Es interesante presentar los nuevos contenidos por medio de apoyo visual: wallchart, flashcards, puppets, etc., de forma contextualizada.
} 
que solo uno o dos presentaban un desempeño elevado, lo que puede ser considerado como un fenómeno aislado.

En el grado 11 la situación fue distinta. Si bien la distribución de los grupos se realizó de la misma manera que en el grado 801 , los estudiantes mostraron una actitud reacia a trabajar en grupo y su desempeño en general fue bastante malo. En comparación con los resultados individuales, los estudiantes participaban más cuando trabajaban de manera individual y su desempeño era considerablemente alto. Un aspecto relevante en ambas situaciones era la concepción que tenían los estudiantes de ambos grados respecto a la adquisición y aprendizaje de una segunda lengua. Para los estudiantes del grado 801 , esos procesos se les facilitaban más de manera grupal porque desde pequeños los docentes fomentaban las actividades grupales y la comunicación entre ellos a través de la segunda lengua (inglés). Esto permitió una mayor adquisición de L2 y se vio reflejado en una mayor integración en el aula. Por otro lado, en el grado 1102, los estudiantes mostraban una mayor actitud individualista, ya que sus docentes de segunda lengua (Inglés) fomentaban el desarrollo individual de las habilidades comunicativas (Escuchar, hablar, leer y escribir). En ellos se fue formando una cultura de individualismo, en la que cada quien sabe que tiene que hacer sin necesidad del otro. En términos de Durkheim, el grado 1102 puede ser considerado como una pequeña sociedad en donde se ve presente la solidaridad mecánica. El grado 801, por lo anteriormente planteado, puede considerarse como un ejemplo de solidaridad orgánica. Se observa, por un lado, una gran presencia de la conciencia colectiva y, por otro, cada miembro posee una parte de los conocimientos generales y sus recursos: todos dependen de todos. Este tipo de solidaridad se suele presentar en las sociedades desarrolladas. 


\section{Conclusiones}

El pensamiento de Durkheim aún continúa muy presente, no solo en el ámbito de la sociología; la educación también fue tema de su interés, al considerarla como un elemento que le permite al individuo integrarse a la sociedad con la principal intención de mejorar su condición humana. La escuela es, junto al hogar, el lugar en donde este proceso se lleva a cabo. La adquisición de una segunda lengua como el inglés puede tomarse como un intento de integración a un tipo de sociedad en la cual, bajo supuestos de progreso establecidos por un sistema social. Es pertinente mencionar aquí que autores como Bourdieu, realizan una crítica al pensamiento funcionalista presente en la obra de Durkheim. Lo señalan de tener un pensamiento que busca la perpetuación de la desigualdad social, que no sólo está ligada al aspecto económico, sino también al aspecto sociocultural. Posteriormente, este proceso se llamaría Capital Cultural, que busca limitar el acceso al conocimiento de acuerdo a los intereses del Estado.

La aplicabilidad de los conceptos de Durkheim, Solidaridad Mecánica y Solidaridad Orgánica, propuestos en su texto "La división de trabajo social en el campo de la educación" es claramente notable. Incluso es posible establecer reflexiones con relación a la adquisición de una segunda lengua (L2), en donde un nivel de solidaridad presente en un aula de clases puede ser tomado como un indicador de integración al sistema social propio, junto al desempeño en actividades que involucran la adquisición esta segunda lengua. La adquisición de una segunda lengua (L2), abordada desde la perspectiva sociocultural de Vygotsky, es asociable claramente a la teoría de Durkheim, en tanto que ambos hablan de la sociedad como un elemento en común que permite el desarrollo del sujeto. Le permite al sujeto adquirir un sistema de códigos (lenguaje) en un contexto específico (lengua) para integrarse posteriormente en cierto nivel a aquel sistema social al que pertenece. Para dar cuenta del nivel de integración medible (síntesis de una concepción psicológica (Vygotsky) y una concepción sociológica (Durkheim), a través del nivel de solidaridad, ya sea orgánica o mecánica presente 
allí, es necesario recurrir a lo que es denominado por Edgar Morin como transdisciplinariedad.

Finalmente, respecto a lo obtenido en el aula de clases, es necesario manifestar que, si bien el nivel de integración que se pudo percibir a través del desarrollo de las distintas actividades, tanto grupales como individuales, permite establecer un diagnóstico respecto al modo en que los estudiantes respondieron. En el grado 801 aparentemente se presentó un mayor desempeño en las actividades grupales que en las individuales (grupos con mayor presencia de solidaridad orgánica, actuando el nivel de integración y de distribución de las distintas labores como indicadores de este fenómeno); la respuesta contraria se observó en el grado 1102. Cabe resaltar que estos resultados pueden ser el resultado de algo más: factores emocionales, carencia de capital cultural o desconocimiento de las temáticas tratadas. También podrían dar respuesta a este fenómeno, factores que pueden ser considerados e incluso estudiados posteriormente, lo que se deja a libre consideración del lector.

\section{Referencias Bibliográficas}

Bourdieu, P. (2005). Capital cultural, escuela y espacio social. Siglo XXI, México. Pp 206.

Bourdieu, P. y J. C. Passeron. (1996). La Reproducción: elementos para una teoría del sistema educativo. Fontamara. México.

Bourdieu, P. y J. C. Passeron. (2004). Los Herederos: los estudiantes y la cultura. Siglo veintiuno editores. Buenos Aires $216 \mathrm{p}$.

Bracho, T. (1990). Capital cultural: impacto en el rezago educativo. Revista Latinoamericana de Estudios Educativos (México), Vol. XX, No. 2, pp. 13-46.

Bruner, J. (1998). Realidad mental y mundos posibles. Barcelona: Gedisa.

Carrizo, Prieto \& Klein. (2004). Transdisciplinariedad y Complejidad en el Análisis Social. Publicado por el Programa MOST, Organización de las Naciones Unidas para la Educación, la Ciencia y la Cultura. 1, rue Miollis, 75732 París Cedex 15, Francia.

Colorado, A. (2009). El capital cultural y otros tipos de capital en la definición de las trayectorias escolares universitarias. Ponencia $X$ congreso nacional de investigación educativa (México). 
De Garay. (2001). Los actores desconocidos. Una aproximación al conocimiento de los estudiantes. Colección Biblioteca de la Educación Superior. México: ANUIES.

De La Herrán, Agustín (2011). Complejidad y Transdisciplinariedad. Revista Educação Skepsis, n. 2.

Durkheim, E. (1973). La división del trabajo social. Schapire Editor SRL.

Durkheim, E. (1975). Educación y sociología. Ediciones Península. Barcelona.

Espinoza, O. (2008). El enfoque del paradigma funcionalista en torno a la naturaleza, alcances, metas y proyecciones del proceso de reforma educacional. Revista Iberoamericana de Educación ISSN: 1681-5653.

Feito, R. (s.f.). Teorías sociológicas de la educación. Universidad Complutense Madrid.

Recuperado

de

http://pendientedemigracion.ucm.es/BUCM/cps/lecturas/4.htm

Gangas, S. (2011). Values, Knowledge and Solidarity: Neglected Convergences Between Émile Durkheim and Max Scheler. Springer Science+Business Media B.V. 2011 DOI 10.1007/s10746-011-9195-8

Lucci, M. (2007). La propuesta de Vygotsky: La psicología sociohistórica. Pontifícia Universidade Católica de São Paulo. Recuperado de http://www.ugr.es/ recfpro/rev102COL2.pdf

Martín, E. (2003). Una crítica de la sociología de la educación crítica. Anduli Revista Andaluza de Ciencias Sociales No 2 / 2003.

Martín, Perez \& Nájera. (2009). Biografía. "Emile Durkheim". Recuperado de http://teoriasocialibero.blogspot.com/2009/09/quien-fue-y-que-hizo-emile-

durkheim.html

Merton, R. (2002). La División Del Trabajo Social de Durkheim. Revista Española de Investigaciones Sociológicas REIS. 201-209. Recuperado de http://www.redalyc.org/articulo.oa?id=99717892009

Morales, L. (2009). Durkheim y Bourdieu: Reflexiones sobre la educación. Rev. Reflexiones 88 (1): 155-162.

Mufioz, C. \& Lopez, D. (2003). Aprendizaje temprano de una segunda lengua. Encuentro Revista de investigación e innovación en la clase de idiomas. Núm 1314. Multiversidad Mundo Real Edgar Morin. (2012). La transdisciplina en la evolución de las disciplinas. Recuperado de http://www.edgarmorin.org/que-estransdisciplinariedad.html

Parsons, T (1971). El salón de clase como sistema social: algunas de sus funciones dentro de la sociedad norteamericana. Publicado en Revista Educación y sociedad $\mathrm{N}^{\circ} \quad 6, \quad$ España. Recuperado de: http://www.google.com.mx/search?hl=es\&lr=lang_es\&rlz=1R2GGLL_es\&q=El+aul $a+$ como + sistema + social $+y+$ parsons\&start $=10 \& s a=N$. 
Prus, R. (2011). Examining Community Life "in the Making”: Emile Durkheim's Moral Education, 56-111. Recuperado de: http://www.jstor.org/stable/41485698

Para citar este artículo:

Piña, D. (2016). Consideraciones sobre la adquisición de L2 a través de la perspectiva sociológica de Emile Durkheim y su obra: la división del trabajo social. Collectivus, Revista de Ciencias Sociales, 3(1), 107-128. doi: http://dx.doi.org/10.15648/Coll.1.2016.6 